\title{
The Effects of Generalized Reciprocal Exchange on the Resilience of Social Networks: An Example from the Prehispanic Mesa Verde Region
}

\section{ROBERT G. REYNOLDS}

Department of Computer Science, Wayne State University, Detroit, MI 48202, USA; Adjunct Research Associate, Museum of Anthropology, University of Michigan, Ann Arbor, Michigan 48104, USA

email:reynolds@cs.wayne.edu

TIMOTHY A. KOHLER

Department of Anthropology, Washington State University, Pullman, WA 99164-4910, USA

email:tako@wsu.edu

ZIAD KOBTI

School of Computer Science, University of Windsor, Windsor, ON, Canada N9B-3P4

email:kobti@uwindsor.ca

\begin{abstract}
The initial version of the model used in this study, Village 1.0, was implemented by Tim Kohler and a team of developers mostly from Washington State University. The original model addressed environmental constraints only and did not attempt to model social interaction. In a recent paper we employed Cultural Algorithms as a framework in which to add selected social considerations. In this paper we extend our previous model by adding the ability of agents to perform symmetrically initiated or asymmetrically initiated generalized reciprocal exchange. We have developed a state model for agents' knowledge and, given agents' different responses based on this knowledge. Experiments have shown that the network structure of the systems without reciprocity was the simplest but least resilient. As we allowed agents more opportunities to exchange resources we produced more complex network structures, larger populations, and more resilient systems. Furthermore, allowing the agents to buffer their requests by using a finite state model improved the relative resilience of these larger systems. Introducing reciprocity that can be triggered by both requestors and donors produced the largest number of successful donations. This represents the synergy produced by using the information from two complementary situations within the network. Thus, the network has more information with which it can work and tended to be more resilient than otherwise.
\end{abstract}

Keywords: cultural algorithm, multi-agent, network resilience, reciprocity, small world networks, archaeology, U.S. Southwest

\section{Introduction}

The initial version of the model used in this study, Village 1.0, was implemented by Tim Kohler and a team of developers from Washington State University (Kohler et al., 2000). The simulation relives the settlement and farming practices of the Pueblo Indians of the Central Mesa Verde region of Southwest Colorado based upon archeological, soils, treering, and other data sources. The objective of the model was to present an approach to 
understanding the behavior of the region's inhabitants, including especially the causes for their periodic aggregation into larger settlements, and the reasons for their eventual emigration from the region. The reasons for the emigration of this population, and the concomitant disappearance of many aspects of their social system, have been key issues in the archaeology of the Americas for many years (Kohler, 2000). Many theories have been proposed in order to solve this "mystery." The approach taken by Kohler and colleagues was to start with the most fundamental of these propositions: climate change.

The "Great Drought" of the late A.D. 1200s and the Little Ice Age (LIA) have both been invoked as reasons for the depopulation of this region and the surrounding area in the thirteenth century. The term LIA refers to "an interval within the last millennium when alpine glaciers in many parts of the world advanced and when climatic conditions were significantly cooler than today" (Van West and Dean, 2000, p. 27). In his original simulation Kohler and his colleagues tested the hypothesis that changes in precipitation affecting the farming potential of this region might, by themselves, produce the depopulation of the region known from the archaeological record. Using paleoproductivity data for the region generated by Van West (1994) that was in turn based on tree-ring proxies for precipitation and local soils data, Kohler and colleagues generated a Swarm-based multi-agent simulation model for land use and population in this area between A.D. 900 and 1300.

Kohler's original simulation suggested that something other than precipitation changes had a hand in the demographic history of the Mesa Verde region, since the model did not generate the expected depopulation of the region. The focus of the original model was solely on farming practices and the distribution of land suitable for farming. The social interactions of individual households (agents) were purposefully omitted in order to see how much of the system's behavior could be explained by changing distributions of rainfall and arable land alone.

Current research involves elaborating this model in several directions. Kohler and colleagues from the Laboratory of Tree-Ring Research of the University of Arizona and from Statistical Research, Inc., of Tucson are attempting to revise the paleoproductivity model to account for changes in temperature and length of growing season on productivity, and to extend the paleoproductivity model back to A.D. 600. A parallel effort is to model the changing availability of groundwater sources in this region between A.D. 600 and 1300 (Kolm and Smith, 2003). Because there is empirical evidence that protein resources and especially big game became limiting on this landscape by the latest occupation (Driver, 2002; Kohler, 2000) we are also beginning to model the availability and use of deer, rabbit, hare, and turkey — the major wild and domesticated animal resources in the region (Johnson, 2003).

Finally, some previous effort has been devoted to understanding the effects on these settlement systems of a one-way "gifting" of maize (Kohler and Yap, 2003). This is an implementation of what Sahlins (1972) has termed "generalized reciprocity" except that gifting is not necessarily directed to close relatives (no kinship was tracked in these simulations) but was directed towards random close neighbors. We noted that such gifting tended to increase the total numbers of households that could be supported on the landscape; that it did not affect the degree of household mobility; and that it tended to reduce the variability of aggregation from one run to the next when these runs started from the same parameter 
settings but were subject to some degree of randomness for initial household placement, initial sizes of households and ages of their members, and various run-time factors in which stochasticity played some role.

In other recent work (e.g., Kobti et al., 2003) we extended Kohler's original model by establishing a framework using Cultural Algorithms in which to embed a population of social agents, and we documented the impact that their social interaction and cultural learning had on the system performance. In particular, we began to be interested in the nature of the social networks that produced by various combinations of social parameters. While the Cultural Algorithm provides a framework in which the agents can learn to select various combinations of co-adapted parameters, our goal now became to see how varying these parameters causes certain aspects of the social networks to appear and/or dissipate.

Specifically, we focused on one parameter, search or move radius. This determines how far away from the original household a new family will settle, or if a family decides to leave its current location how far away it can look for a new one. We view the move radius as a surrogate measure of social aggregation. Thus, when a new agent household is formed or relocated, its position is governed by the range over which it can search for another position. Links are then made between the household and the households of its relatives. We assume that these links represent communication links between agents that are necessary to coordinate their activities. We then wish to observe the social structures that emerge from the interaction of the agents and their environment.

We began by looking at the tradeoffs between the tendencies for social aggregation on the one hand and the environmental constraints that tend to force dispersal on the other (Kobti et al., 2003). In the simulation, the agents effectively tried to strike a "balance" or "find a centering" between the social forces for aggregation and the environmental forces in the region. This notion of finding a balance or center is a common theme in Puebloan cultures (Thompson, 2002). We demonstrated that the agents tended to form "small world networks" based upon the kin relations that we had added to Kohler's original model, and that these network structures can be differentially impacted by environmental perturbations.

However, no explicit movement of information, or resources within the network was considered. In the present paper we extend our model of the agent household to include a preliminary state model for each household agent. This state model is the basis upon which various models of reciprocal exchange among the individuals within the network are produced. This will allow the flow of resources to move between individuals having different states, for example between those that have excess productivity and those in need of food. We provide three different frameworks for such flows. In the first case, those who are in need ask their relatives for assistance. In the second case, individuals with excess food poll the nearby relatives to see if they need assistance. The third approach combines both of the activities within the same network. It is suggested that the combined approach synergistically enhances the resilience of the network.

In Section 2 we briefly overview the Cultural Algorithm framework in which the model is embedded. Then in Section 3 we describe how the social networks are modeled. In Section 4 we focus on describing the state model of an agent's behavior. Section 5 presents the basic simulation system used here. The results of our experiments are given in Section 6, and we summarize what we have learned in Section 7. 


\section{The Cultural Algorithm Framework}

Holland developed a formal framework for any generic adaptive system (Holland, 1975). His framework for adaptation concerns a system that is able to alter its structure and/or behavior based on experience in some set of performance environments (Reynolds, 1979). Adaptability is the capacity to function in an uncertain or unknown environment, and to use information to evolve and learn (Conrad, 1983). Adaptation can take place at three different levels: the population, individual and component level (Angeline, 1995).

Cultural Algorithms consist of a social population and a belief space (Reynolds, 1979) as shown in figure 1. Selected individuals from the population space contribute to cultural knowledge by means of the acceptance function. The cultural knowledge resides in the belief space where it is stored and updated based on individual experiences and their successes or failures. In turn, the cultural knowledge controls the evolution of the population by means of an influence function. A Cultural Algorithm thereby provides a framework in which to accumulate and communicate knowledge so as to allow self-adaptation in an evolving model (Flannery et al., 1989).

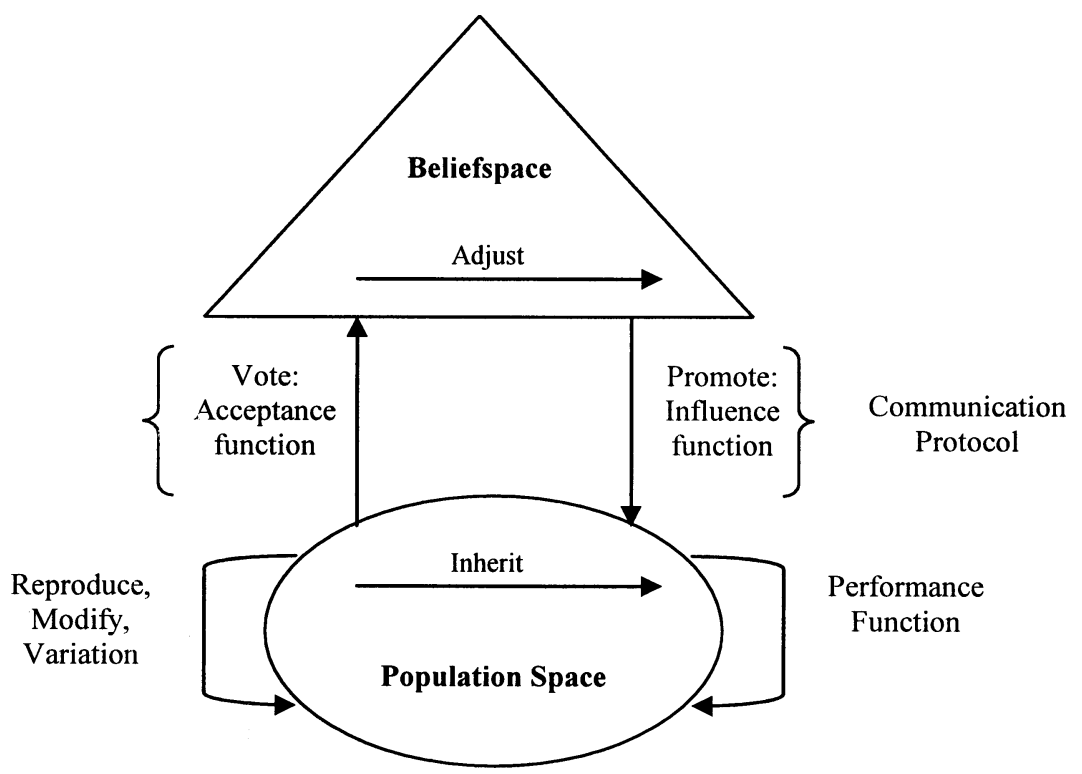

Figure 1. Cultural algorithm framework.

There are at least five basic categories of cultural knowledge that are important in the belief space of any cultural evolution model: situational, normative, topographic, historical or temporal, and domain knowledge (Reynolds, 04/2003). In our cultural model all of these knowledge sources are present as well. For example, in our current model we assume that agents will have access to knowledge about the distribution of agricultural land (topographic knowledge), the distribution of rainfall as it occurs over time to the extent that it affects agricultural production (history or temporal knowledge), and concerning agricultural planting 
and harvesting techniques (domain knowledge). These three knowledge sources are fixed at this time.

We will concentrate on the acquisition or learning of just two types of knowledge by agents at this point, situational and normative knowledge. Situational knowledge is a "snapshot" of the state of the world. The world can be viewed as a sequence of situations linked by social behaviors. Examples of specific individual experiences correspond to a set of situational knowledge or relationships between individuals and their physical and social environments. Normative knowledge on the other hand describes how a rational agent should act in terms of ranges of socially acceptable behavior (Russell and Norvig, 1995). In other words, normative knowledge defines a standard or ideal that can be used to judge which behavior is desirable or undesirable (Valente and Brueker, 1994). For example, in our scenario agents can learn in general which kin relations are most likely to respond to requests for resources; and on the other hand, which kinship relationships are most likely to require aid. This knowledge can reduce the search effort of individuals and improve the flow of resources within the system. Figure 2 illustrates the representation of sample normative knowledge in the belief space within a Cultural Algorithm framework.

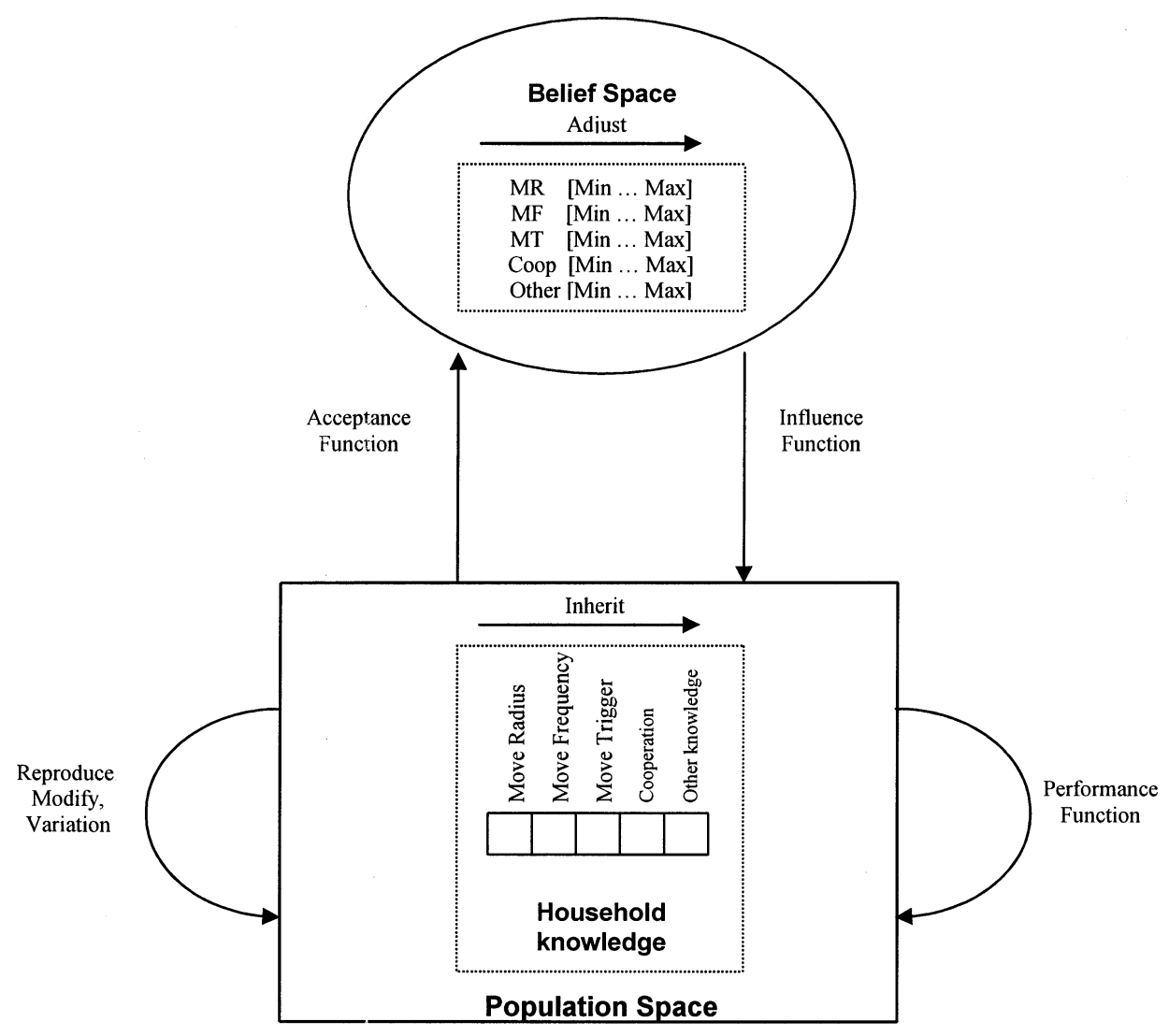

Figure 2. The cultural algorithm framework with normative knowledge. 


\section{Modeling the Social Networks}

The first step in developing social relationships between households is to develop the social network through which these relationships can be expressed. In this model the basic relationship is based on kinship and the strength of the relationship is impacted by the distance between agents that share a kinship relationship between them. Here, each agent is a household composed of a husband, a wife and their children. Household members live together in the same location, share their agricultural production, and are affected by the same environmental conditions. Children can grow up, marry, and move out to form their own households. Their connections to their parent households and siblings are maintained in our model. Similarly, the parents maintain ties to their children. When one of the parents in a household dies, the other can form a new household with an available single agent. The initial structure of the social network here supports the notions of parents, siblings, and grandparents on both sides of the family.

The layout of the social network from the perspective of a household is given in Table 1.

Table 1. Connected nodes identified by the kinship social network.

\begin{tabular}{ll}
\hline $\begin{array}{l}\text { ParentHHTagA } \\
\text { ParentHHTagB }\end{array}$ & $\begin{array}{l}\text { a link to the parent from the mother's side, } \\
\text { a link to the parent from the father's side, } \\
\text { onildHHTag }\end{array}$ \\
helink to each child that moves away from this \\
household and form its own household, \\
one link to each extended family member.
\end{tabular}

Each household (agent) is identified by a unique tag in the system. One can think of this tag as the household's name that uniquely identifies it. In the initial step, agents are formed without any links (or relations). In subsequent annual steps an agent updates its links based on a set of simple rules: (1) If a new child marries and moves out on its own, the parent household will then have a link to it. The child household in turn will maintain a link to the parent household which it just moved away from. This effort is duplicated since it is assumed that each child comes from a different parent household. So cumulatively, a new household will have links to two parent households as a minimum; one link to the parent household from the wife's side while the other is to the parent household from the husband's side. (2) A child household can also remember its siblings. They are immediate relatives and hence the child will have links to them. (3) A household member can remarry if the spouse dies. In that case, the household would keep a link to the initial household, except now as a relative, and updates a new parent link to the new spouse's household. (4) Kinship relations rules can be extended to other family members. (5) Other concepts such as friendships and neighbors can also be modeled, which are of course based on absence of kinship relations, but also taking into account, for example, a household's location and its proximity to other households. The overall social network is maintained dynamically and updated every time step. 


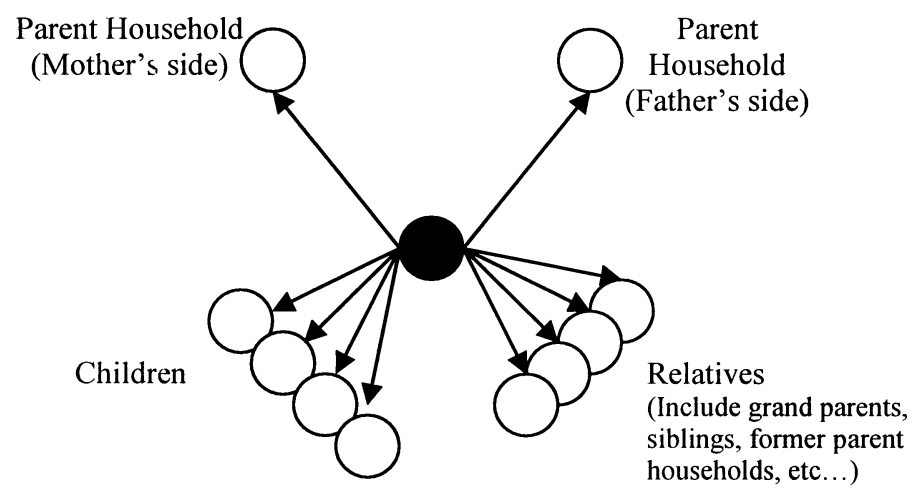

Figure 3. Individual node and the representation of its links.

The set of kinship relations between agents cumulatively forms a directed graph. Each time step, the current graph is stored in the form of an adjacency list. Then we can plot the graph and examine the distribution of structural properties for the vertices (agents) and their edges (relations) as shown below in Table 2.

Table 2. Social network structure as defined by the kinship relations.

\begin{tabular}{ll}
\hline TAG & Agent Tag whose links we are describing \\
X, Y & Position of this agent in the world \\
ParentTagA, ParentTagB & Links to each parent's Tag (-1 means no link) \\
ChildHHTag $_{1} \ldots$ ChildHHTag $_{c}$ & Link to each child \\
RelativeHHTag $_{1} \ldots$ RelativeHHTag $_{2}$ & Link to each relative \\
\hline
\end{tabular}

The format of the output file containing the adjacency list generated for one time step (each year) is:

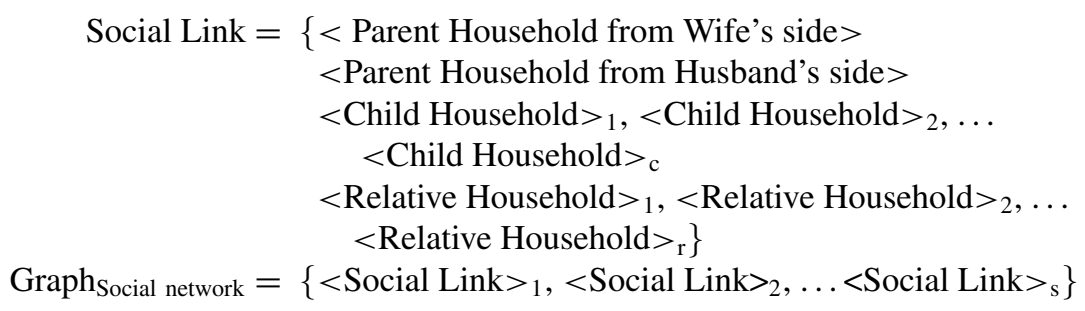

This kinship model sets the stage for the flow of materials between agents. In particular, we assume a custom of generalized reciprocity that is practiced only between kin. Such practices are common in many societies (Flannery et al., 1989; Sahlins 1972, pp. 191-275). We will investigate how such reciprocity shapes the demography, settlement system, and the resultant social networks in the region, and how, in turn, those networks are subsequently 
affected by the results of climatic change, such as a drought. Droughts of varying severity and length occur several times during the 400 years we simulate here. How we model reciprocity is the subject of the next section.

\section{Cooperation Framework}

Three strategies for reciprocal aid were explored and compared with the case in which no goods are exchanged between agents. Table 3 lists the methods of exchange used and gives a brief description of each. Reciprocal exchange is defined in terms of the exchange of maize between agents related through kinship. Unlike trade between agents, the model of generalized reciprocity used does not keep a record of debts owed by particular agents. Modeled after the compassionate and human response of social beings, agents may seek to ask their relatives for food in a time of need, while others donate their surplus to their relatives during prosperous times. In other words the exchange is activated by the requestor, the donor, or both. Each version is potentially reciprocal; the only difference is in terms of who provides the information that triggers the exchange. The current approach to exchange implements a more refined version of Sahlins' (1972) "generalized reciprocity" than that represented previously by Kohler and Yap (2003), since the exchanges here are indeed limited to kin, and present as possibilities both asymmetric and symmetric exchanges (Table 3). Our use of these terms focuses on whether an exchange can be initiated only by the donor or alternatively only by the receiver ("asymmetric") or by either ("symmetric").

Table 3. Description of the different cooperation methods at the kinship level.

\begin{tabular}{ll}
\hline Cooperation method & Description \\
\hline 0 & No cooperation. No exchange of food between households. \\
1 & $\begin{array}{c}\text { When an agent requires food, it is allowed to select and request food } \\
\text { from within its kinship network in order to survive. } \\
\text { When an agent has excess food, above a determined threshold } \\
\text { amount, it is allowed to select an individual(s) } \\
\text { from its kinship network and donate some of its excess. } \\
3\end{array}$ \\
& Both methods 1 and 2 are enabled simultaneously. \\
\hline
\end{tabular}

\subsection{Individual Agent Strategy}

Each agent's basic strategy is to farm, harvest and store enough maize to survive. In a time of drought or on lands of relatively low yields, the household may not have enough food to sustain it during a given year. Starvation in a non-cooperative model directly can cause the death of the entire household. In order to avert this, the starving household can seek the help of another household known to it from its kin network. The selection method for a prospective donor can be one of several types, random, fitness proportional, and round robin. However, the model is flexible enough to allow other strategies to be implemented in the future. 
If the selected agent is in the appropriate state then the exchange of resources is performed if they have sufficient resources to fill the entire request. If an agent is not able to fulfill the entire request, the requesting agent again can choose to make another attempt to request the needed amount of food to survive. The number of attempts that can be made by an agent in a year is another model parameter.

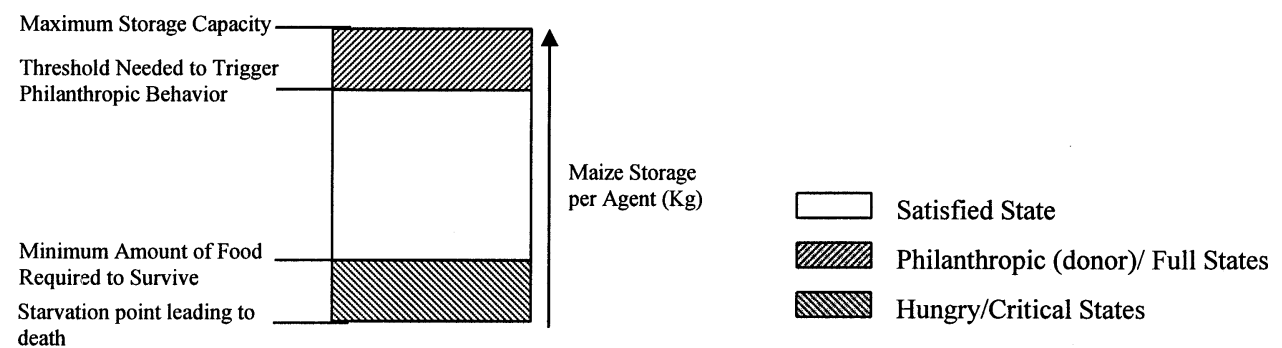

Figure 4. Actual maize amount in storage determines the state of an agent.

The basic states that agents can be in are given in figure 4. An agent can have more food stored than needed and can therefore be a donor. An agent can have more than the minimum but less than what is needed to be a donor. Likewise an agent can have fewer calories than needed, the hungry state. Finally, the food level for an agent can be less than the starvation point, the critical state. They can ask for and possibly receive food from a donor. There are three cases of exchange based upon the states of the individuals. In the first case, an individual who is in either a hungry or critical state can ask for resources from a kin-related agent who is in the donor state. In the second method, an individual agent who is in the donor state can provide resources to kin who are in the hungry or critical states. In the third case, reciprocal aid can be triggered either by agents who are in the hungry or critical state and by agents who are in the donor state.

In either case of symmetric reciprocal exchange, a limiting constraint is the physical distance between the two agents. For instance an agent cannot request food from another agent who is beyond its immediate search area. A similar case applies for an agent donating some of its surplus. A search-radius distance parameter is implemented in each of the two cooperation strategies similar to the move-radius parameter.

\subsection{Selection Methods}

A number of possible household methods for the selection of a related household to exchange with are implemented in the system. The methods present in the current model are: random, roulette-wheel or fitness-proportional selection, and round-robin selection. In general, an agent can be allowed to adopt any approach to select another agent to cooperate with it. The three different choices are given in Table 4 below. In this paper, we fix the selection scheme to be random. An agent can randomly select the agent it wants to cooperate with as long as this agent is within its cooperation radius and is in the appropriate state. If cooperation is initiated by the requestor then the requestor must be in a state of need and must ask for a 
donation from an agent in a state of excess. Likewise, if the donor initiates the cooperation, then the donor must be in a state of excess and must select an agent in a state of need. Figure 5 describes the other state-based agent interactions supported within the current model.

Table 4. List of the implemented selection methods.

Random
$\begin{aligned} & \text { Randomly choose an agent from the kinship network within a given range. } \\ & \text { Roulette }\end{aligned}$
$\begin{aligned} & \text { Start with random selection of agent (equal initial wheel portion) from the } \\ & \text { kinship network, then reward or penalize the probability of selecting this } \\ & \text { agent depending on whether the agent cooperated with or declined the request. }\end{aligned}$
Round Robin $\quad \begin{aligned} & \text { All agents that are kin with the agents and that are } \\ & \text { in the given cooperative radius are systematically each given a turn. }\end{aligned}$

\subsection{A State-Based Model of Agent Behavior}

The approach used to implement the symmetric reciprocity strategy used here is based upon a set of states that an agent can take. The state of an agent can change dynamically each year based upon the food produced and consumed by the agent (maize here). An agent normally acquires its food from harvesting its plots of land. The yields are directly dependent on the environmental factors affecting the plot yield as well as the farming practices and the labor force available within a household.

How an agent participates in the exchange process depends on its current state. For instance, an agent that is doing well may choose to donate some of its excess food or may choose to keep it to itself in store. At the other end of the spectrum, an agent straining under drought conditions and bad crop yields is in constant need of support. With a cooperative social network, the agent has the opportunity to tap into the wealth of its nearby relatives and ask for food in order to survive.

The state transition diagram for each agent is given in figure 5. Arcs between nodes represent state changes that can take place as the result of crop yields and reciprocal exchange. The arc that points from the state back to itself represents the fact that the agent can change its state or remain in that state from year to year.

The states are as follows:

Satisfied -An agent is in a "satisfied" state when it has sufficient food in storage to feed the entire family in the household.

Philanthropic - An agent becomes a philanthropist when it has a surplus of food in storage, defined in terms of stored maize in excess of a given threshold. For instance, an agent stocking $90 \%$ or more of its storage capacity would be able to donate its surplus food to others.

Hungry - A buffer state is implemented at the level just above critical need so that the agent can try to prevent the starvation associated with critical need. When the agent is left with its last food ration then it enters a "hungry" state that triggers precautionary requests for food to avoid starvation. 
Critical—An agent that has insufficient or no food to eat has no choice but to ask for food or face starvation and imminent death. If the household does not receive its ration to feed the entire family it will die.

Death-An agent is marked for immediate removal from the system.

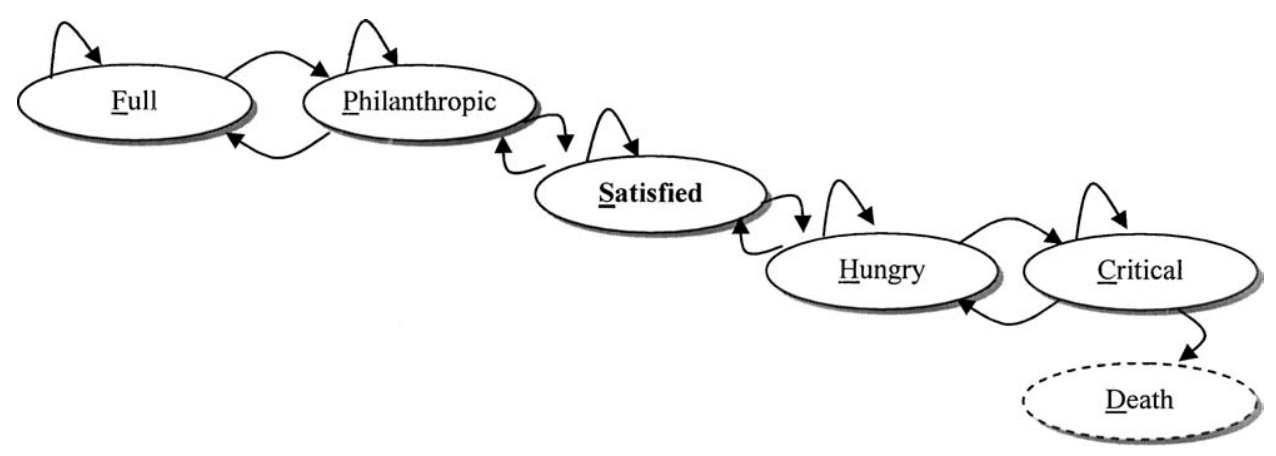

Figure 5. Agent State Transition Diagram. Note that additional states transitions are possible directly between F, P, S, H and C states, but the ones shown are the most frequent.

A state interaction matrix shown in Table 5 allows us to examine the cooperative relationships that are possible between two individual agent households based upon their respective states.

Table 5. Matrix of possible interactions between two agents A and B- Agent A depending on its current state will interact with Agent B differently depending on its own current state. (N/E = No Exchange).

\begin{tabular}{lcccc}
\hline & \multicolumn{4}{c}{ Agent B } \\
\cline { 2 - 5 } Agent A & Philanthropic & Satisfied & Hungry & Critical \\
\hline Philanthropic & N/E & P donates to S & P donates to H & P donates to C \\
Satisfied & N/E & N/E & N/E & N/E \\
Hungry & H requests from P & H requests from S & H requests from H & H requests from C \\
Critical & C requests from P & C requests from S & C requests from H & C requests from C \\
\hline
\end{tabular}

\section{Experimental Framework}

The Cultural Algorithm framework outlined in Section 2 is implemented here in the Swarm simulation environment (Langton, 1995). This environment provides a framework within which to facilitate development and experimentation with a large number of agents in a dynamic environment. Currently the system is written entirely in Objective-C and uses the Swarm 2.2 pretest libraries. The model is a graphical multi-agent simulation that allows us to probe individual agents in a dynamic environment. Agents reside in a cellular space that corresponds to our study area of $1500-\mathrm{km}^{2}$ with a spatial resolution of $200 \times 200 \mathrm{~m}$ in the 
Four Corners region. At every model step in the simulation, the potential paleoproductivity for dry farming of each cell is updated using a database ultimately depending on tree-ring data (Van West, 1994). Agents are observed by means of probes that examine their internal properties or by accumulating output files for later examinations.

Here, the simulation was run over the period from A.D. 900 to 1281 for each combination of constraints. For each time step the network is generated and stored in a file named "links $<$ YEAR $>$.out". This file is used to examine the properties of the emerging network and any characteristic that we find relevant to network resiliency. Visualization of the network is done using MatLab or a Visual Basic viewer (shown here in figure 6). The program we developed allows us to plot the graph and to closely examine the distribution and densities of the links between agents. As the graph becomes more and more dense, especially as agents develop more social links over time, we can visually filter out nodes with smaller number of links and display only those edges attached to a node with a certain associated out-degree. In other words, we can identify the agents with the highest connectivity in the social network in terms of the number of associated links that they maintain.

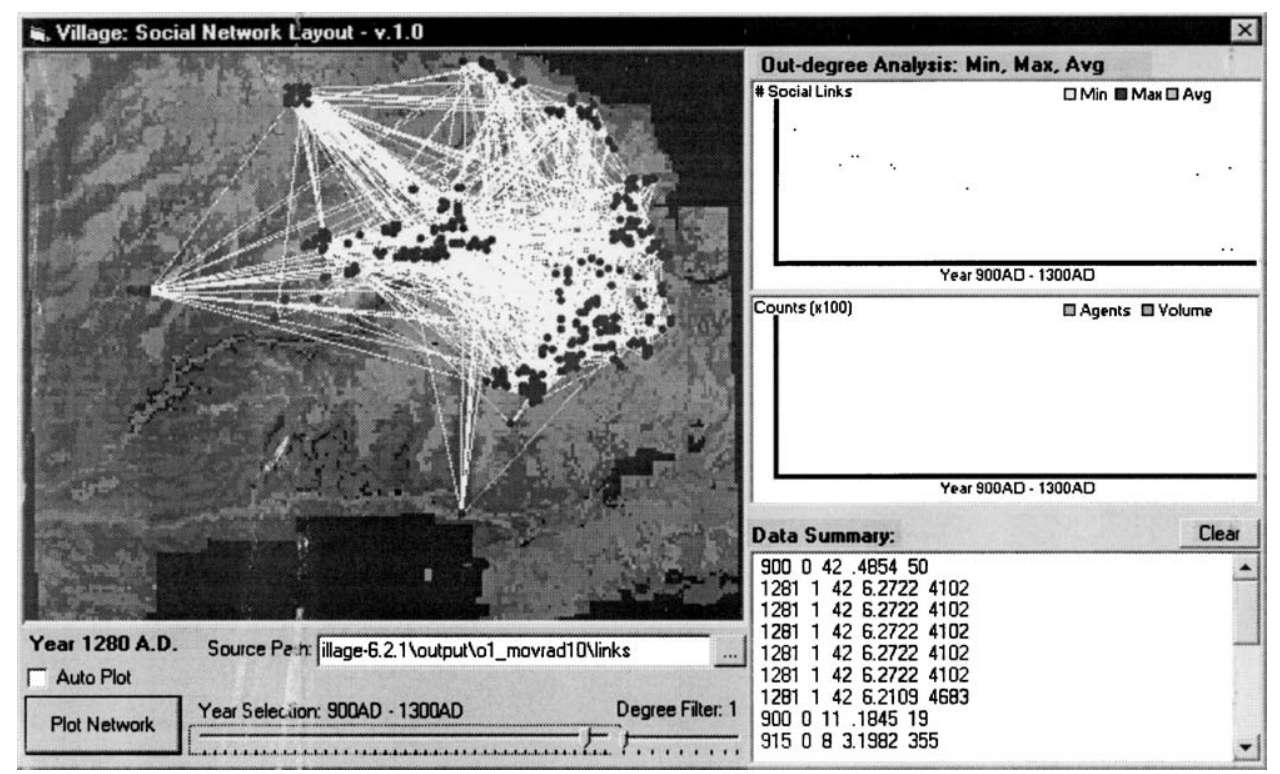

Figure 6. Using a search radius of 10 (MOV_RAD 10) the network is plotted for A.D. 1280 showing highly dense nodes.

In the experiments we conducted, the search radius that the agent uses when looking for a new location to move to, or an agent with whom to exchange resources, was varied from 5 to 30 pixels (each pixel is $200 \mathrm{~m}$ on a side) and movement was subject to prior land-use constraints. For the different values of the move radius tested, the volume of the social network and the number of network links over time were generated. The volume is the sum of all of the links associated with each individual household (agent) present. The idea here 
is that with a larger move radius the agents can relocate farther away from where they were located before. Since relocation occurs frequently during periods of population growth, a larger move radius means that they relocate farther away from a previously successful area.

\section{Results}

To test the various effects of cooperation under the changing environmental conditions built into the model, the fixed rules for reciprocity described earlier in the state diagram are implemented in order to guide the strategic behavior of the agent. In each run, the same fixed paleoproductivity pattern is used. This pattern is based upon tree-ring data (Van West, 1994) and an impression of its changing mean and variability through time can be gained from figure 7 (Van West and Dean, 2000), which is however considerably smoothed. Notice that the rainfall pattern exhibits substantial variability over the 400-year period which will put some stress on the system's resilience, or ability to bounce back following perturbation. The fluctuations in agricultural productivity in the model are not known in advance by the agents.

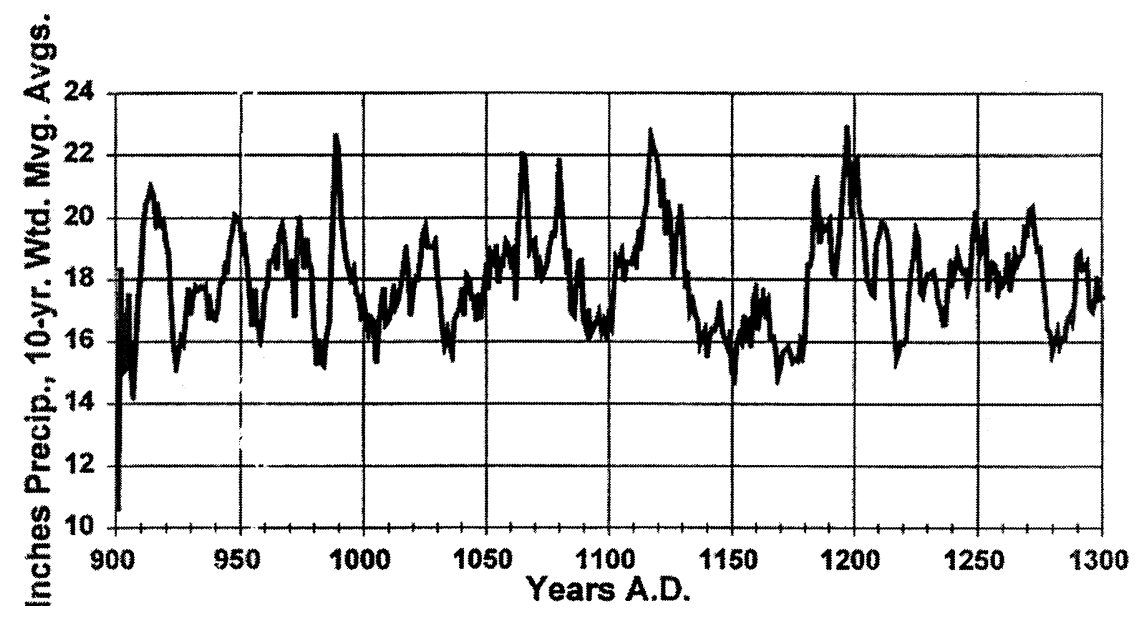

Figure 7. Reconstructed total annual precipitation (inches) estimated from the Mesa Verde Douglas-fir chronology.

The three possible protocols for exchange discussed earlier were examined using a set of experiments: the first involves an agent requesting food; the second involves an agent donating food; and the third approach combines the effect of the first two.

In Tables 6 through 8 the various sets of experimental configuration are given. Each row is a run configuration which was run 5 times. So, a total of 105 runs were made. Each configuration is specified in terms of a number of constants. Cooperation type denotes the type of reciprocity protocol used; 0 represents no exchange; 1 represents that the recipient initiated requests only; 2 represents donor initiated requests only; and 3 allows both donor and recipient to initialize requests. Move radius constrains the distance over which an agent 
can relocate and exchange resources. The next four columns place constraints on who can request and who can give along with who can donate and who they can donate to. The state abbreviations are: $\mathrm{F}=$ Full; $\mathrm{P}=$ Philanthropist; $\mathrm{S}=$ Satisfied; $\mathrm{H}=$ Hungry; $\mathrm{C}=$ Critical; (and $\mathrm{D}=$ Dead).

To begin, in Table 6, we ran the previous model with no reciprocity, COOP TYPE $=$ 0 , for move radii of 10,20 and 30 pixels respectively. There are no constraints on the interaction of agents; '-' represents none, since there is no interaction.

\begin{tabular}{lccccc} 
Table 6. & Experimental baseline control-No cooperation. \\
\hline Coop type & Move radius & Donor & Requestor & Donate to & Request from \\
\hline 0 & 10 & - & - & - & - \\
0 & 20 & - & - & - & - \\
0 & 30 & - & - & - & - \\
\hline
\end{tabular}

Table 7 gives configurations which allow some flow of resources but only when initiated by agents who are in the extremes of the state hierarchy; individuals who have as much as possible (full) or those who need as much as possible (critical). Thus requests in type 1 are only made by agents who are in a critical state. In type 2 , donations are made only by those in the full state. In type 3, both full donors and critical recipients can initiate flows.

An important aspect of the cooperative strategies examined is the potential to allow the agents the ability to maintain a safety "buffer" in their food-storage levels. This safety buffer allows an agent to compute whether it is about to eat its last food ration. This measure is dependent mainly on the make-up of the household members and their food requirements. Currently, the system defines the hungry state as when an agent has only one food ration left to eat. After this is consumed, it moves into the critical state where it must seek food from others. One set of experiments tested the scenarios with the presence of such buffering in anticipation of allowing the agent to foresee and bail itself out of starvation, while another set tested the scenarios without the presence of buffering.

Table 7. Experimental setup without buffering.

\begin{tabular}{lccccc}
\hline Coop type & Move radius & Donor & Requestor & Donate to & Request from \\
\hline 1 & 10 & F & C & CHS & FPS \\
2 & 10 & F & C & CHS & FPS \\
3 & 10 & F & C & CHS & FPS \\
1 & 20 & F & C & CHS & FPS \\
2 & 20 & F & C & CHS & FPS \\
3 & 20 & F & C & CHS & FPS \\
1 & 30 & F & C & CHS & FPS \\
2 & 30 & F & C & CHS & FPS \\
3 & 30 & F & C & CHS & FPS \\
\hline
\end{tabular}


In all of the experiments in Table 7, requestors can request for any agent who is not in need (F, P, and S), and donors can give to any agent who does not have a surplus (C, H and S).

Table 8 relaxes the constraints on the requestor and donor agents. That is, a requestor can be in either a critical or needy state $(\mathrm{C}$ or $\mathrm{H})$. The requestor can be in either a full or partially full state (F or P). The constraints of donations to, and requests from are the same as in Table 7. These experiments allow the system to "buffer" the effects of the extreme states by allowing both donations and requests to occur before the extreme states are reached.

Table 8. Experimental setup with buffering.

\begin{tabular}{lccccc}
\hline Coop type & Move radius & Donor & Requestor & Donate to & Request from \\
\hline 1 & 10 & FP & CH & CHS & FPS \\
2 & 10 & FP & CH & CHS & FPS \\
3 & 10 & FP & CH & CHS & FPS \\
1 & 20 & FP & CH & CHS & FPS \\
2 & 20 & FP & CH & CHS & FPS \\
3 & 20 & FP & CH & CHS & FPS \\
1 & 30 & FP & CH & CHS & FPS \\
2 & 30 & FP & CH & CHS & FPS \\
3 & 30 & FP & CH & CHS & FPS \\
\hline Legend: F= Full; P $=$ Philanthropist; S Satisfied; H Hungry; C Critical; (D = Dead).
\end{tabular}

So we can now examine how network resiliency and structure is affected as we gradually relax our constraints on resource exchange.

For each configuration, data were collected on the agents' response over time, the number of requests, the number of fulfilled requests, the number of donations available and accepted, and the total amounts of food changing hands each year. The circumstances under which a request might be refused include the bounding limitations of the cooperation radius, a probability for cooperation to take place, and whether or not an agent has already reached full capacity in the case of a full state. The emergent social network is also examined in terms of its standard properties such as average, maximum and minimum arcs forming hub node densities, and any observable collapse of major nodes. The number of surviving agents is also recorded yearly to examine the effects of environmental variability on population size in the presence of cooperation in its different forms and in its absence.

\subsection{The Impact of Reciprocity on Network Resiliency}

While runs were done for each combination of states for each of the three different move radii, only the results for move-radius 30 are given in this section. Figure 8 gives three 
figures that summarize the results of 5 simulation runs in which no gifting occurs between the agents. The agent interaction produces kinship networks over time but the networks are not used to provide possibly symmetric reciprocal aid to kin explicitly. Figure 8(a) gives the maximum, minimum, and average number of links between the agents in the emergent social network. The average number of links is 6 , very characteristic of a small world network. Notice that although the average and minimum number of links per household are not affected by changes in precipitation over time, the maximum number of links per node is. The nodes with a large number of links are called the "hub" nodes. The hub nodes provide the connectivity required for an individual agent to search the network. Figures 8(b) and 8 (c) give the volume of the social network over time while figure 8 (c) gives the number of active households (agents). The number of agents goes down slightly around the year 1140 as drought conditions begin. Overall, the resultant networks are the least complex of the three sets of experiments, but the most resilient to change. The droughts that occur near the end of the period and the increased variability do not impact the structure of the network very much.

Social Network Accumulated by Agents Per Year (Kinship) (COOP 0, MOV_RAD 30)

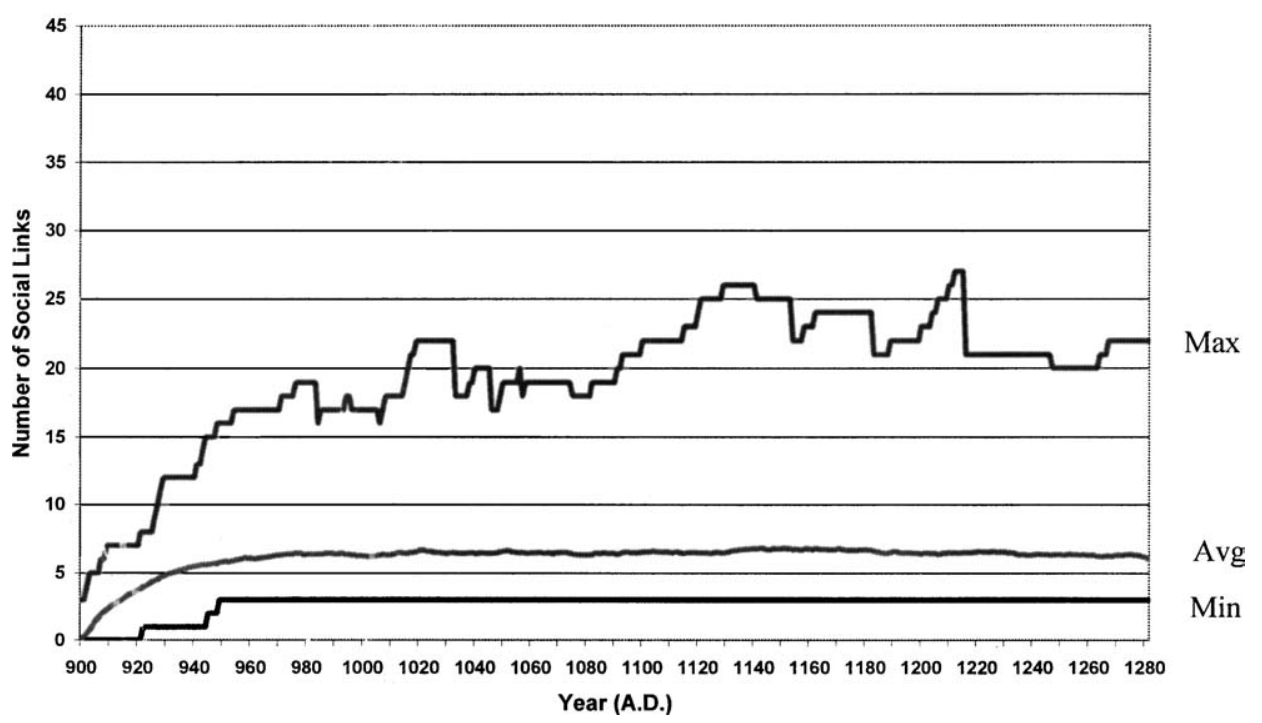

(a)

Figure 8. (a)-(c): No reciprocity across the network. (Coop 0). 


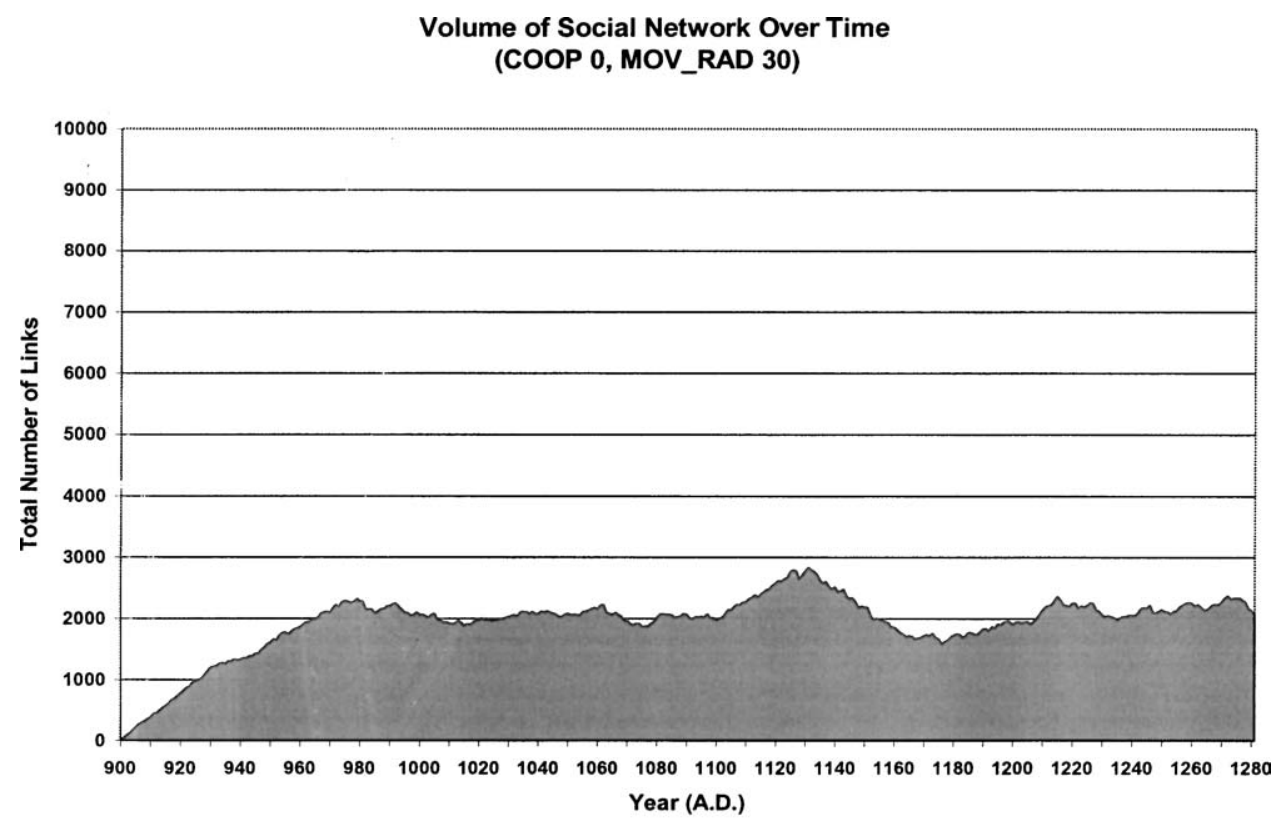

(b)

Agent Population Counts Over Time (Number of Living Households) (COOP 0, MOV_RAD 30)

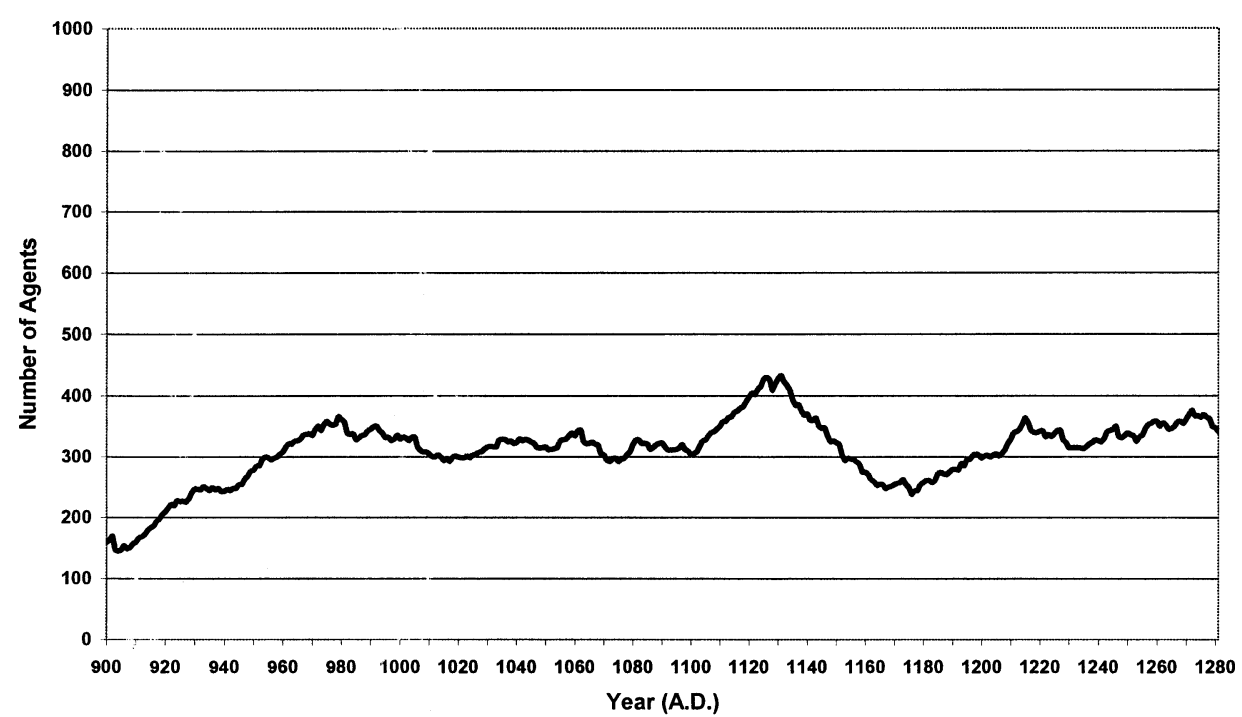

(c)

Figure 8. (Continued). 
When requestor-initiated reciprocity is allowed, the number of agents, the link volume, and the maximum number of links all increase over the type 0 case as shown in figure 9. Figure 9(a)-(c) give the results when only agents in critical need (c) can request only from those who are full or partially full $(\mathrm{F}$, and P). Figure 9(d)-(f) gives the results produced when any agent in need $(\mathrm{C}$ and $\mathrm{H})$ can make a request.
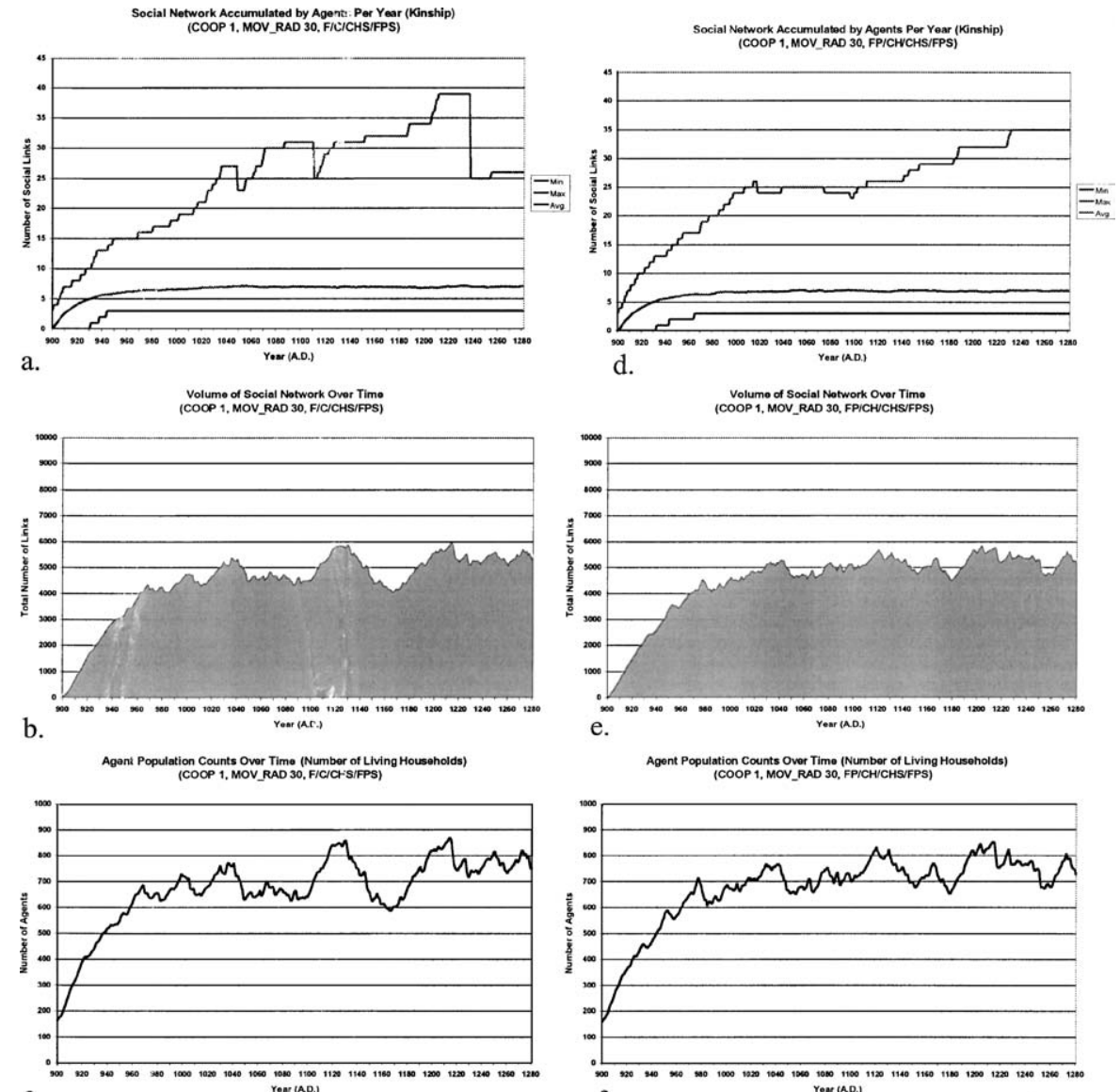

c.

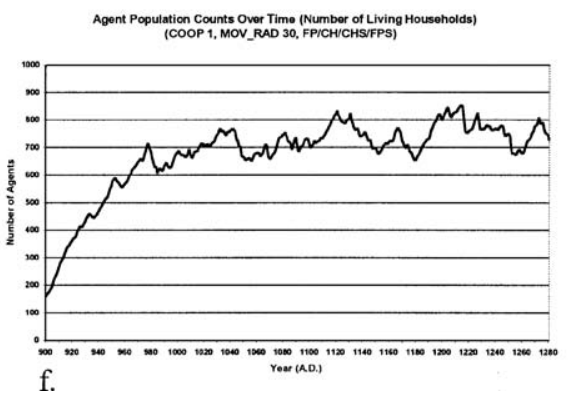

Figure 9. Coop 1 (Request initiated reciprocity) Network volume and agent counts over time. (a)-(c): Left column. (d)-(f): Right column.

The addition of reciprocity increases the complexity of the system by producing larger and more complex networks; these networks are much more variable in response to the same environmental input. For example, the maximum number of links shown in 8 (a) varies markedly throughout the run but particularly near the end as does the social network volume and the total number of households. What is interesting is while both the buffered and non-buffered cases of reciprocity are more complex than the no-exchange case: the buffered version has more uniform variability and slightly larger peak values for household 
number, link volume, and maximum link number. Thus, adding in the buffering capability dampens the fluctuations produced by only reacting to the extreme states while maintaining an increased complexity over the no-exchange case.

Figure 10 gives results from the set of experiments that deal with donor-initiated reciprocity. As before, figures 10(a)-(c) describe the unbuffered case where only agents in a full state are able to donate to their kin while figure 10 (d)-(f) describe the case in which a donor is anyone not in need. Notice that the complexity of the emergent social network in terms of its number of agents, social volume, and maximum size of hub nodes is not as great as that produced when the exchange in only initiated by the receiver. This is because the donors in this case distribute their resources randomly and are not able to focus their donations where they are most needed. This may change when cultural knowledge is allowed. Thus, allowing the donor to initiate the exchange identifies where in the network the excess is, but may not allow it to get to those in critical need.
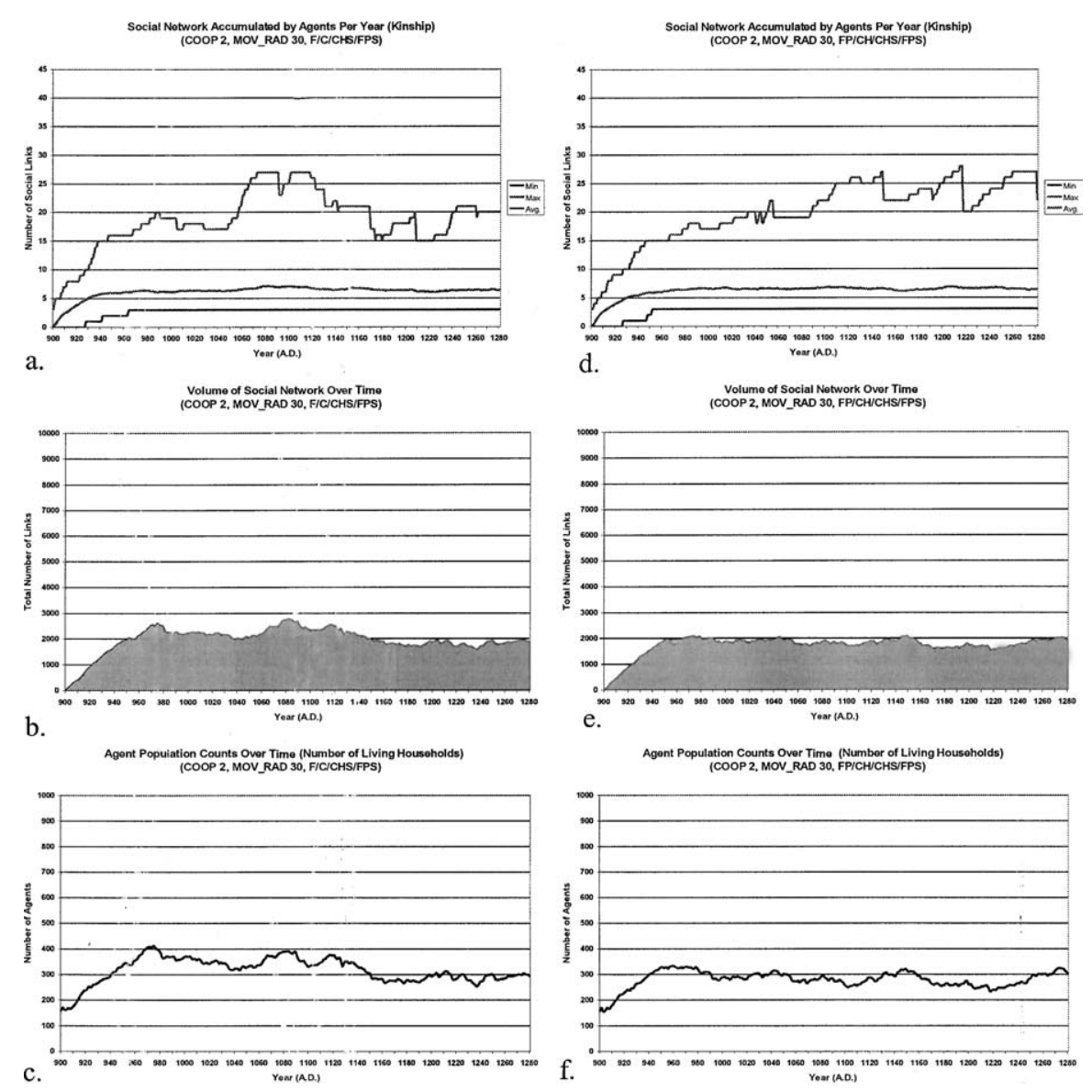

Figure 10. Coop 2 Network volume and agent counts over time. (a)-(c): Left column. (d)-(f): Right column. 
In fact, there are several things to note so far:

1. Buffering significantly reduces variability in household number, maximum link size, and social volume in both the donor-initiated and the requestor-initiated exchange;

2. Requestor-initiated exchange tends to produce more complex networks in terms of volume than donor-initiated exchange.

3. Requestor-initiated exchange also produces more variability in network complexity with respect to volume than donor-initiated exchange.

a.

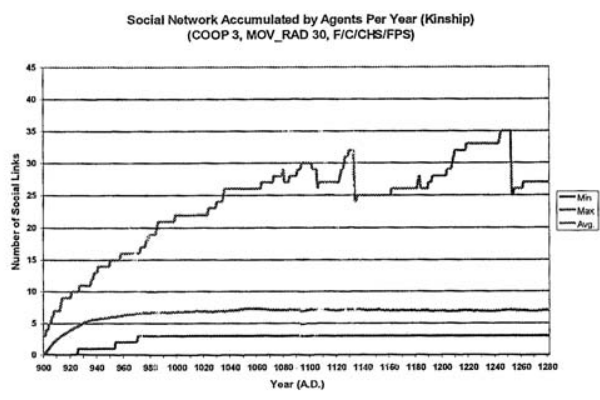

Volume of Social Notwork Over Time
(COOP 3, MOV_RAD 30, FICICHSIFPS)

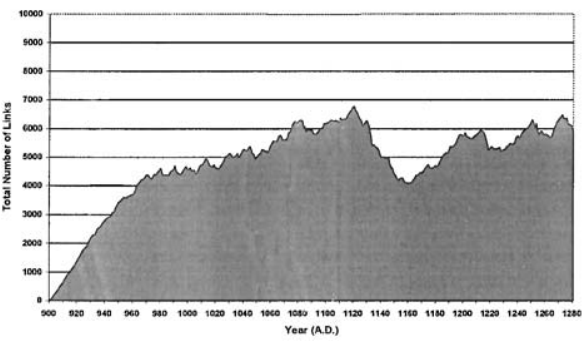

b.

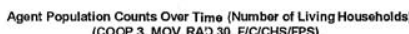

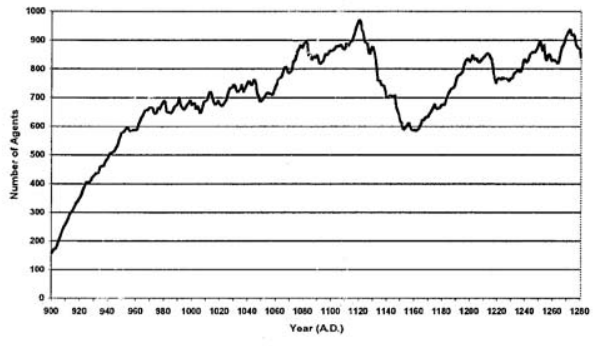

d.

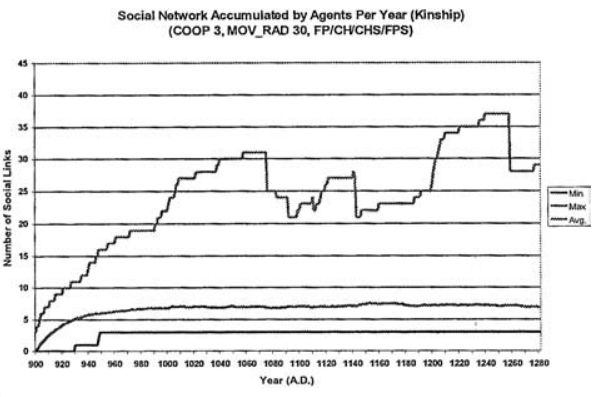

Volumo of Social Notwork Ovor Time
COOP 3, MOV RAD 30, FPICHICHSIFPS

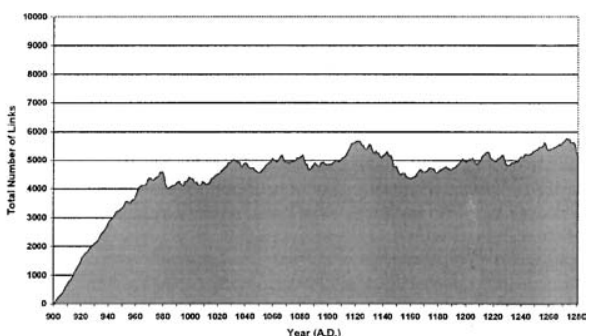

e.

Agont Population Counts Ovor Time (Number of Living Housoholds)
(COOP 3, MOV RAD 30, FPICH/CHSIFPS)

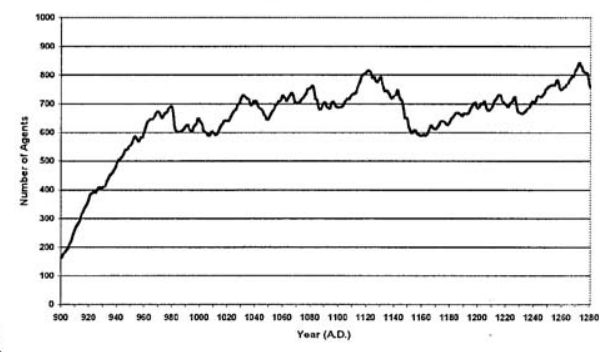

Figure 11. Coop 3 Network volume and agent counts over time. (Unbuffered: (a)-(c): Left column; Buffered: (d)-(f): Right column). 
It seems as though there may be potential complementarities between the two approaches to the initiation of exchange. The requestor-initiated approaches produced larger populations in both the critical and buffered case than the donor-initiated approaches but at the cost of increased variability in the wake of environmental change.

Figure 11 shows the synergy that is produced by combining both the requestor-initiated and the donor-initiated modes of reciprocal exchange in both the buffered and unbuffered cases for the combined system and for the networks that are produced. The unbuffered system however exhibits larger fluctuation in social volume, household numbers, and maximum links. Adding the buffering capability to the system generated a slight decrease in complexity and produced a more stable system.

One of the interesting results that we see is that by adding reciprocity and buffering to the system we effectively produce more complex social structures characterized by larger population densities, larger network volumes and larger hub complexity. The next question is whether these more complicated social structures are more or less resilient than those of systems without exchange? An answer to this can be seen in Table 9, a graphical display

Table 9. Correlations between reconstructed total annual precipitation and kinship exchange experiments.

\begin{tabular}{lcccc}
\hline & & & \multicolumn{2}{c}{ Moving average 10 years } \\
\cline { 4 - 5 } Experiment number & Coop type, MOV_RAD & Buffering type & Raw correlation & Absolute \\
\hline 1 & C0R10 & & -0.37537499 & 0.375375 \\
2 & C0R20 & & 0.099921433 & 0.099921 \\
3 & C0R30 & & -0.176052643 & 0.176053 \\
4 & C1R10 & FC & -0.053460942 & 0.053461 \\
5 & C1R20 & FC & -0.100295947 & 0.100296 \\
6 & C1R30 & FC & -0.16650569 & 0.166506 \\
7 & C1R10 & FPCH & 0.065355737 & 0.065356 \\
8 & C1R20 & FPCH & -0.078928888 & 0.078929 \\
9 & C1R30 & FPCH & 0.087028229 & 0.087028 \\
10 & C2R10 & FC & 0.022800943 & 0.022801 \\
11 & C2R20 & FC & 0.053468116 & 0.053468 \\
12 & C2R30 & FC & -0.142869376 & 0.142869 \\
13 & C2R10 & FPCH & 0.063688484 & 0.063688 \\
14 & C2R20 & FPCH & 0.110300349 & 0.1103 \\
15 & C2R30 & FPCH & 0.080030221 & 0.08003 \\
16 & C3R10 & FC & -0.048454564 & 0.048455 \\
17 & C3R20 & FC & 0.020085898 & 0.020086 \\
18 & C3R30 & FC & 0.028298228 & 0.028298 \\
19 & C3R10 & FPCH & -0.044933474 & 0.044933 \\
20 & C3R20 & FPCH & 0.006129205 & 0.006129 \\
21 & C3R30 & FPCH & 0.042143731 & 0.042144 \\
\hline & & & &
\end{tabular}




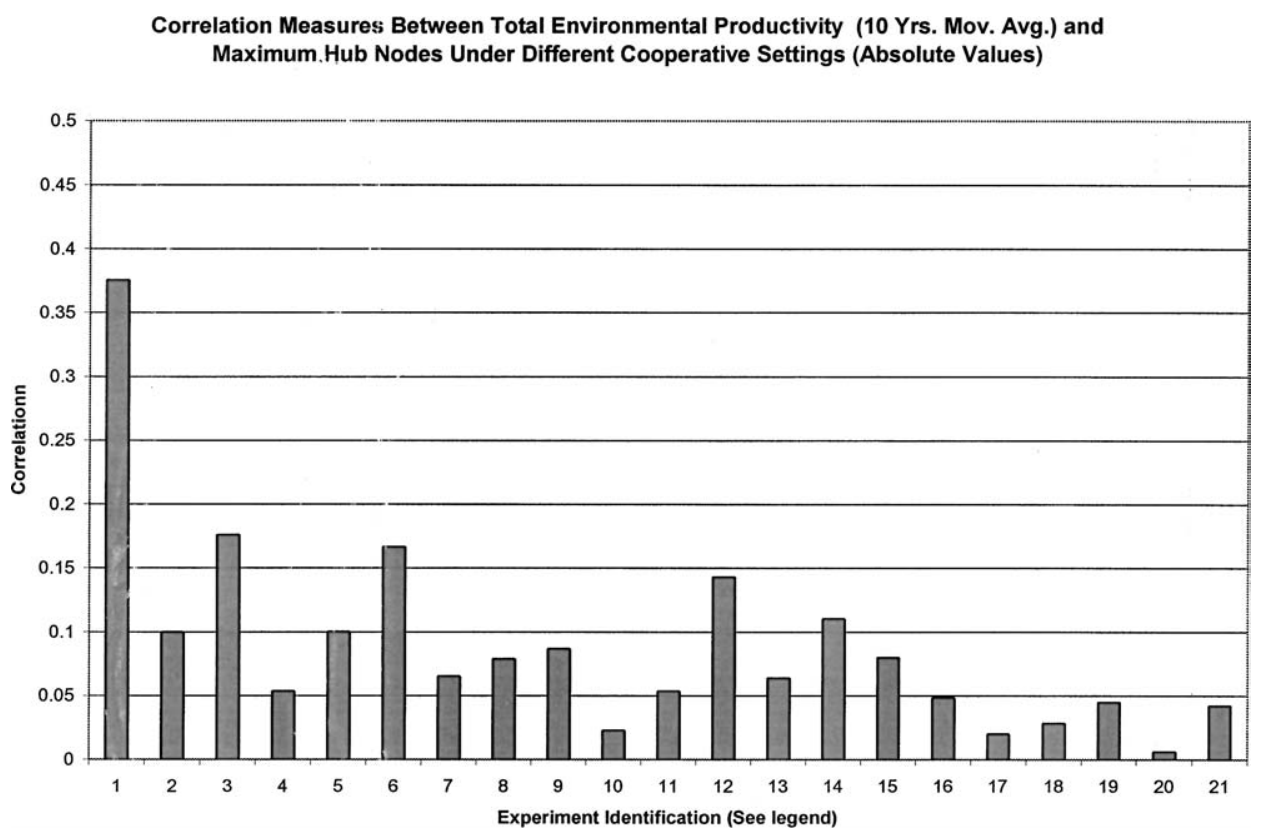

Figure 12. Absolute values of correlation trends shown for Experiments 1 to 21 as defined in Table 9.

is also given in figure 12, where we compute the correlation between productivity and the maximum hub size between A.D. 900 and 1300 in the valley. The correlation between maximum hub size and the tree-ring based precipitation index was computed using 10year moving averages for each. Here, the more resilient the system, the less the correlation between the total annual precipitation and the social structure. That is, in a resilient system, change in average rainfall has a reduced impact on the social structure than a less resilient system.

If we compare the correlations for the system with no exchange (COR10-C0R30) against that for the system with recipient and donor initiated exchange plus buffering it is clear that the latter system even with a larger population is more resilient than the former for each of the three move radii in terms of the maximum hub size.

Figures 13 and 14 demonstrate specifically why the combined scheme produces the largest and most resilient exchange networks. In figure 13, we give the number of successful donations produced by the four different exchange combinations for each of the three different search radii tested. The figures show that as the move radius increases, the volume of the donations decreases. There are two basic reasons for this. On the one hand, increased move radius might improve productivity so less exchange is needed. On the other hand, an increased move radius allows individuals to be spread out farther from each other. So, there are likely to be less donors and requestors nearby to exchange with each other. An agent in the current model is limited to one random attempt to find an exchange partner. With larger move radius, the exchange is less likely to occur. 

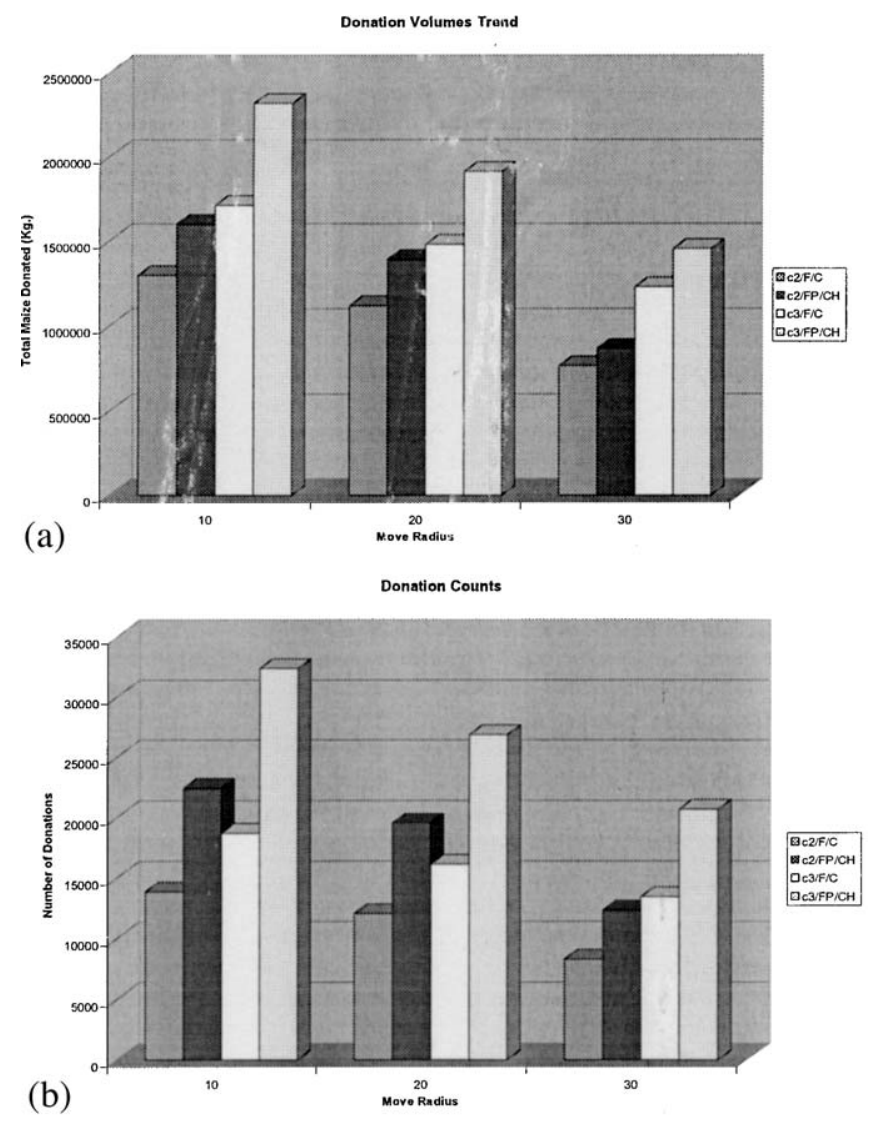

Figure 13. (a) The amount of donated maize $(\mathrm{kg})$ over the 400 year period for each move radius $(10,20,30)$ and each of four cooperation types (C2 without buffering, $\mathrm{C} 2$ with buffering, $\mathrm{C} 3$ without buffering, and $\mathrm{C} 3$ with buffering). Donation volumes increase as move radius decreases, as buffering is added, and when both donors and requestors are active. (b) Total counts of total successful donations over the 400 year period for each move radius $(10,20,30)$ and each of four cooperation types (C2 without buffering, $\mathrm{C} 2$ with buffering, $\mathrm{C} 3$ without buffering, and $\mathrm{C} 3$ with buffering). The number of successful donations increases with decreased move radius, the addition of buffering, and when both donors and requestors are active.

Within any given radius, the buffered solutions outperformed the un-buffered ones. In figure 14, we also see that the number of requests for each exchange configuration decreases as the move radius increases for the same reasons as above. The fact that there is little difference between the number of requests in each configuration reflects the fact that the same environmental perturbations are being presented in each case and create a similar need per agent. However, the fact that the configurations differ in terms of the number of donations made reflects the ability of the system to opportunistically deal with shortfalls. 


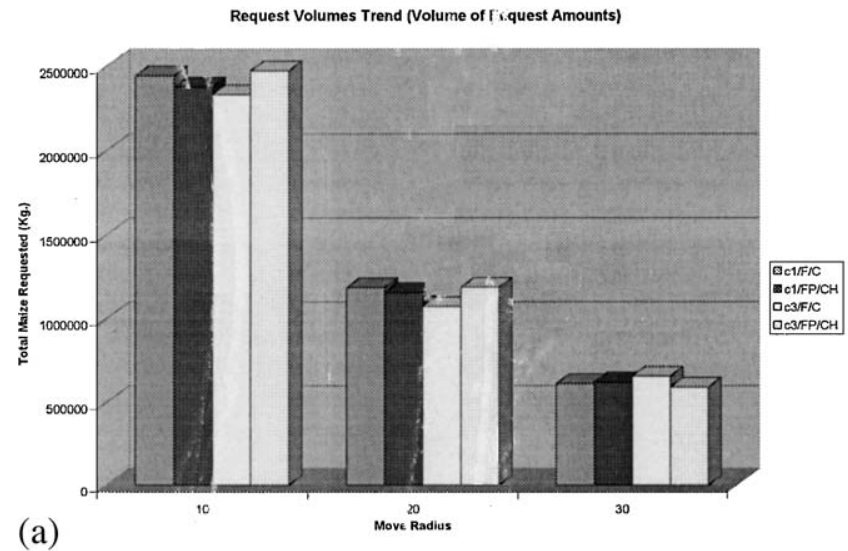

(a)

Number of Requests $/$ ide

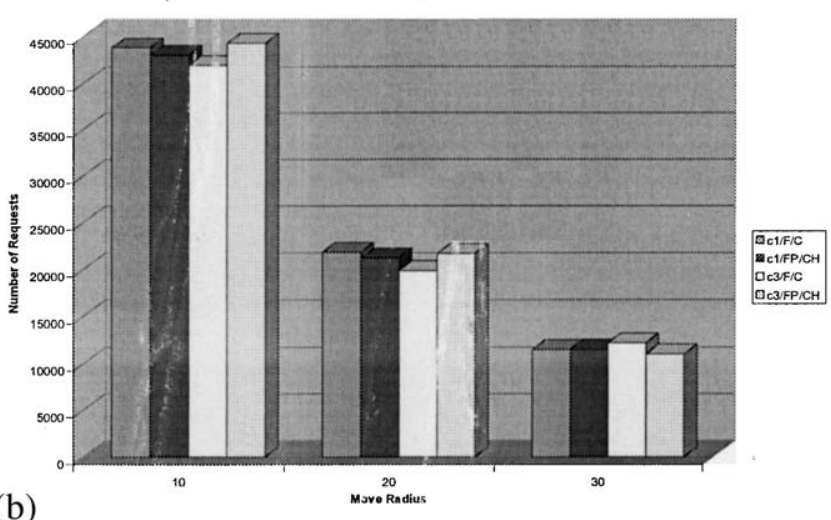

Figure 14. (a) The volume of requested maize ( $\mathrm{kg}$ ) over the 400 year period for each move radius $(10,20,30)$ and each of four cooperation types ( $\mathrm{C} 1$ without buffering, $\mathrm{C} 1$ with buffering, $\mathrm{C} 3$ without buffering, and $\mathrm{C} 3$ with buffering). The requested volume increases as move radius decreases. Here buffering or having both requestors and donors active does not increase performance substantially. (b) The total number of requests over the 400 year period for each move radius $(10,20,30)$ and each of four cooperation types ( $\mathrm{C} 1$ without buffering, $\mathrm{C} 1$ with buffering, $\mathrm{C} 3$ without buffering, and $\mathrm{C} 3$ with buffering). The number of total requests increases as move radius decreases. Here buffering or having both requestors and donors active does not increase performance substantially. (c) The volume of requested maize actually received $(\mathrm{kg})$ over the 400 year period for each move radius $(10,20,30)$ and each of four cooperation types ( $\mathrm{C} 1$ without buffering, $\mathrm{C} 1$ with buffering, $\mathrm{C} 3$ without buffering, and $\mathrm{C} 3$ with buffering). The volume of maize actually recieved $(\mathrm{kg})$ increases as move radius decreases. Here buffering or having both requestors and donors active does not increase performance substantially. (d) The number of successfully answered requests over the 400 year period for each move radius $(10,20,30)$ and each of four cooperation types (C1 without buffering, $\mathrm{C} 1$ with buffering, $\mathrm{C} 3$ without buffering, and $\mathrm{C} 3$ with buffering). The number of successfully answered requests increases as move radius decreases. Here buffering or having both requestors and donors active does not change performance substantially. 


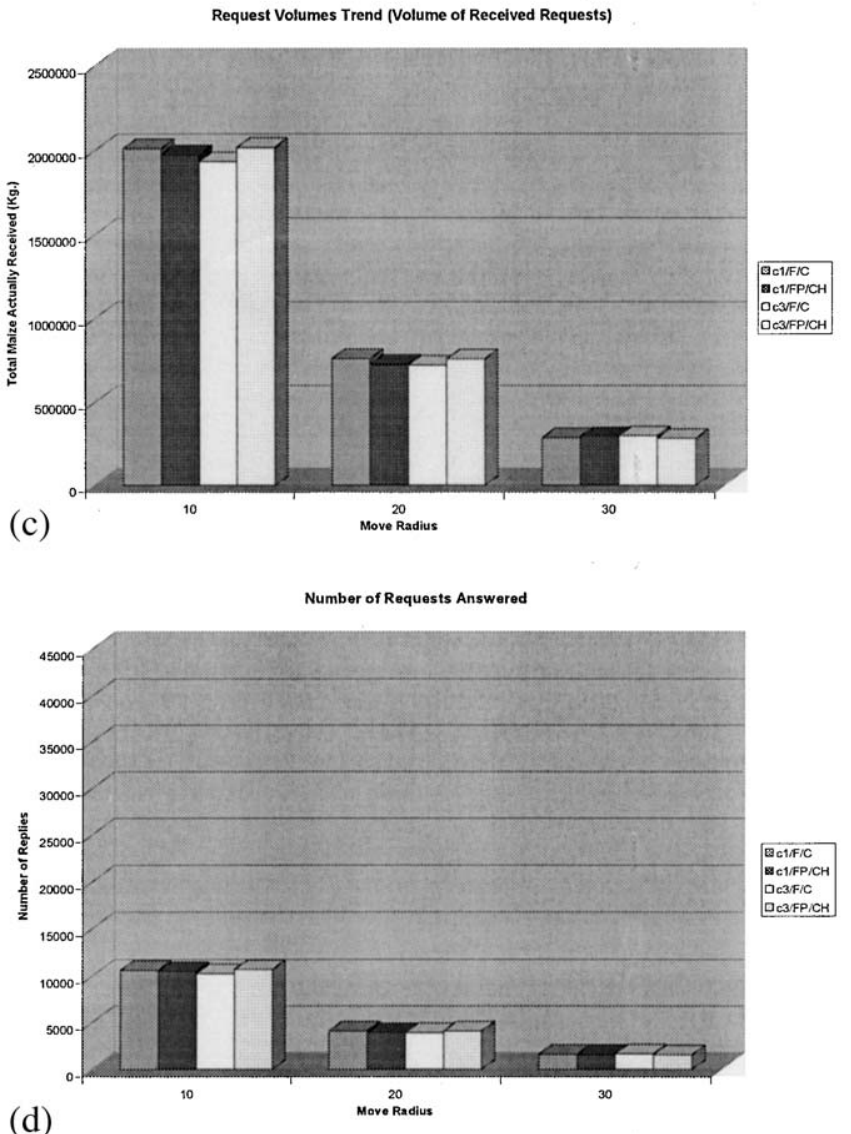

Figure 14. (Continued).

It is also interesting to note the combined donor-recipient approach is always less correlated with environmental change than the no-exchange model regardless of buffering or radius size used.

\section{Conclusions and Future Work}

In this paper we have extended our previous model by adding in the ability of agents to perform exchange of either a symmetric (reciprocal) or asymmetric sort. We have developed a state-based model of agent intelligence and agents can exchange resources based on their current states and that of the environment. Experimental results suggest that the structure of the social network for the system with no or reduced reciprocity (donor or requester only) is simpler than that with full reciprocal exchange (both) but less resilient with regards to changes in precipitation over time than when full reciprocal exchange takes 
place. When agents are given more opportunities to exchange resources across the kinship network they produced more complex network structures. Consequently this led to larger populations and more resilient systems. In addition, when agents were allowed to buffer their requests by using a finite state model we noted improved relative resilience in these larger systems. The introduction of reciprocity that can be triggered by both requestors and donors produced the largest number of successful donations. This represents the synergy produced by using the information from two complementary situations within the network. Therefore, the network has more information with which it can work and tended to be more resilient than otherwise.

Within this framework, agents can learn to generate plans involving a variety of social activity. For example, currently agents randomly select individuals for interaction within the given radius. Our next step will be to allow cultural learning to take place by allowing individual agents to learn who to ask. This can result in cultural knowledge that may help make requests more successful. In other words, can social learning "boost" the ability of the system to efficiently redirect resources where they are needed? This will allow us to examine how much of the variability in system response can be removed by knowledgeable giving and receiving of resources. In the future we want to integrate exchange into a larger repertoire of agent activities using the cultural algorithm framework.

\section{References}

Angeline, P.A. (1995), “Adaptive and Self-Adaptive Evolutionary Computation,” in Marimuthu Palaniswami et al. (Eds.) Communication Intelligence, New York: IEEE Press, pp. 152-163.

Driver, J.C. (2002), "Faunal Variation and Change in the Northern San Juan Region," in Mark D. Varien and Richard H. Wilshusen (Eds.) Seeking the Center Place: Archaeology and Ancient Communities in the Mesa Verde Region. Salt Lake City: University of Utah Press, pp. 143-169.

Flannery, K., J. Marcus and R.G. Reynolds (1989), The Flocks of the Wamani. Academic Press.

Holland, J.H. (1975), Adaptation in Natural and Artificial Systems, 1st edition. Ann Arbor: The University of Michigan Press, 1975; First MIT Press edition. 1992.

Johnson, C.D. (2003), "Modeling Critical Resources: Natural Production and Human-Induced Degradation in the Prehistoric Mesa Verde Region," Paper presented at the 68th Annual Meeting of the Society for American Archaeology, Milwaukee, Wisconsin.

Kobti, Z., R. Reynolds and T. Kohler (2003), A Multi-Agent Simulation Using Cultural Algorithms: The Effect of Culture on the Resilience of Social Systems. IEEE Congress on Evolutionary Computation, Canberra, Australia (to appear).

Kohler, T.A. (2000), "The Final 400 Years of Pre-Hispanic Agricultural Society in the Mesa Verde Region,” Kiva, V. 66, pp. 191-264.

Kohler, T.A., Kresl, James, Van West, Carla, Carr, Eric and Wilshusen, Richard (2000), "Be There Then: A Modeling Approach to Settlement Determinants and Spatial Efficiency among late Ancestral Pueblo Populations of the Mesa Verde Region, U.S. Southwest," in Timothy A. Kohler and George Gumerman (Eds.) Dynamics in Human and Primate Societies: Agent-Based Modeling of Social and Spatial Processes, Santa Fe Institute and Oxford University Press. pp. 145-178.

Kohler, T.A. and L. Yap (2003), "Modeling Reciprocal Exchange in Southwestern Societies." Paper presented at the 68th Annual Meeting of the Society for American Archaeology, Milwaukee, Wisconsin.

Kolm, K.E. and S. Smith (2003), "Modeling Paleohydrology for Settlement Systems in the Canyon of the Ancients Region, Southwestern Colorado," Paper presented at the 68th Annual Meeting of the Society for American Archaeology, Milwaukee, Wisconsin.

Langton, C. et al. (1995), "The Swarm Simulation System A Tool for Studying Complex Systems," Santa Fe Institute. 
Reynolds, R.G. (1979), "An Adaptive Computer Model of the Evolution of Agriculture for Hunter-Gatherers in the Valley of Oaxaca, Mexico," Doctoral dissertation, University of Michigan, Ann Arbor.

Reynolds, R.G. (1999), "An Overview of Cultural Algorithms", Advances in Evolutionary Computation, McGraw Hill Press.

Reynolds, R., J. Brewster and R. Iacoban (2003), "Cultural Swarms: The Impact of Culture on Social Interaction and Problem Solving," in Proceedings of IEEE Swarm Intelligence Symposium, Indianapolis Indiana.

Reynolds, R. and Z. Kobti (2003), "The Effect of Environmental Variability on the Resilience of Social Networks: An Example Using the Mesa Verde Pueblo Culture.” Paper presented at the 68th Annual Meeting of the Society for American Archaeology, Milwaukee, Wisconsin.

Reynolds, R. and Z. Kobti (2003), "A Multi-Agent Simulation Using Cultural Algorithms: The Effect of Culture on the Resilience of Social Systems", Santa Fe Institute Workshop on Human Environmental Interaction, May $16-18$.

Reynolds, R., T. Kohler and Z. Kobti (2003), Paper presented at the workshop on Robustness in Coupled Natural and Human System, Santa Fe Institute, May.

Russel, S. and P. Norvig (1995), Artificial Intelligence-A Modern Approach. Englewood Cliffs, NJ, Prentice Hall. Sahlins, M. (1972), Stone Age Economics. Aldine-Atherton, Chicago.

Thompson, I. (2002), "Native American Perspectives on Sand Canyon Pueblo and Other Ancestral Sites," in Mark D. Varien and Richard H. Wilshusen (Eds.) Seeking the Center Place: Archaeology and Ancient Communities in the Mesa Verde Region, Salt Lake City: University of Utah Press, pp. 257-262.

Valente A. and J. Breuker (1994), "A Commonsense Formalization of Normative Systems," in Proceedings of the ECAI'94 Workshop on Artificial Normative Reasoning.

Van West, C. (1994), Modeling Prehistoric Agricultural Productivity in Southwestern Colorado: A GIS Approach. Department of Anthropology Reports of Investigations 67. Pullman, WA: Washington State University.

Van West, C. and J.S. Dean (2000), "Environmental Characteristics of the A.D. 900-1300 Period in the Central Mesa Verde Region”, Kiva, V. 66, pp. 19-44.

Robert G. Reynolds received his Ph.D. degree in Computer Science, specializing in Artificial Intelligence, in 1979 from the University of Michigan, Ann Arbor. He is currently a professor of computer science and director of the Artificial Intelligence Laboratory at Wayne State University. He is also an Adjunct Associate Research Scientist with the Museum of Anthropology at the University of Michigan, Ann Arbor. His interests are in the development computational models of cultural evolution for use in the simulation of complex organizations. Dr. Reynolds developed a framework, Cultural Algorithms, in which to express and computationally test various theories of social evolution using multi-agent simulation models. He has applied these techniques to problems concerning the origins of the state in the Valley of Oaxaca, Mexico (with Kent Flannery), and the origins of language and culture (with Robert Whallon). He has co-authored two books, Flocks of the Wamani (1989, Academic Press), with Joyce Marcus and Kent V. Flannery, and The Acquisition of Software Engineering Knowledge (2003, Academic Press) with George Cowan. He has received funding from both government (NSF) and industry (Ford Motor Company) to support his work. He specifically thanks the National Science Foundation (BCS-0119981) for the support of this project.

Timothy A. Kohler is an archaeologist and professor in the Department of Anthropology, Washington State University, Pullman. He specializes in Neolithic societies with a focus on the U.S. Southwest. His recent books include The Archaeology of Bandelier National Monument: Village Formation on the Pajarito Plateau, New Mexico (University of New Mexico Press, 2004) and (with George J. Gumerman) Dynamics in Human and Primate Societies: Agent-based Modeling of Social and Spatial Processes (Oxford University Press, 2000). He thanks the National Science Foundation (BCS-0119981) for support of this project.

Ziad Kobti is a Ph.D. student in the Department of Computer Science at Wayne State University. He received his B.Sc. Honors with a double major in Biological and Computer Sciences and an M.Sc. in Computer Science from the University of Windsor (1999). He is a Limited Term Computer Science Lecturer at the University of 
Windsor (2001-) and active researcher at Wayne State University. Currently he is working with Dr. Robert G. Reynolds on developing Cultural Algorithms to add a social intelligence framework to the Mesa Verde Village project. Previous industry experience since 1993 includes a national award-winning critical-time client/server and distributed database software solution programmer for the emergency freight trucking industry, government funded civil and environmental engineering software interface programmer, technical educator/trainer in college and industry, corporate web presence and e-commerce developer, and IT consultant. 